\title{
Augmentation Pressure Point P2
}

National Cancer Institute

\section{Source}

National Cancer Institute. Augmentation Pressure Point P2. NCI Thesaurus. Code

C122086.

The second aortic systolic pressure peak, also referred to as a returning pressure wave, used to calculate the aortic augmentation index. 